\title{
Correlated D-meson decays competing against thermal QGP dilepton radiation
}

\author{
Thomas Lang* \\ Frankfurt Institute for Advanced Studies (FIAS) \\ E-mail: lang@th.physik.uni-frankfurt.de \\ Hendrik van Hees \\ Frankfurt Institute for Advanced Studies (FIAS) \\ E-mail: hees@th.physik. uni-frankfurt.de \\ Jan Steinheimer \\ Lawrence Berkeley National Laboratory \\ E-mail: jsfroschauerelbl.gov
}

\section{Marcus Bleicher}

Frankfurt Institute for Advanced Studies (FIAS)

E-mail: bleicherath.physik.uni-frankfurt.de

The QGP that might be created in ultrarelativistic heavy-ion collisions is expected to radiate thermal dilepton radiation. However, this thermal dilepton radiation interferes with dileptons originating from hadron decays. In the invariant mass region between the $\phi$ and $J / \psi$ peak $\left(1 \mathrm{GeV} \lesssim M_{\ell^{+} \ell^{-}} \lesssim 3 \mathrm{GeV}\right)$ the most substantial background of hadron decays originates from correlated DD-meson decays. We evaluate this background using a Langevin simulation for charm quarks. As background medium we utilize the well-tested UrQMD-hybrid model. The required drag and diffusion coefficients are taken from a resonance approach. The decoupling of the charm quarks from the hot medium is performed at a temperature of $130 \mathrm{MeV}$ and as hadronization mechanism a coalescence approach is chosen. This model for charm quark interactions with the medium has already been successfully applied to the study of the medium modification and the elliptic flow at FAIR, RHIC and LHC energies. In this proceeding we present our results for the dilepton radiation from correlated DD̄ decays at RHIC energy in comparison to PHENIX measurements in the invariant mass range between 1 and $3 \mathrm{GeV}$ using different interaction scenarios. These results can be utilized to estimate the thermal QGP radiation.

8th International Workshop on Critical Point and Onset of Deconfinement

March 11-15

Napa, California, USA

\footnotetext{
* Speaker.
} 


\section{Introduction and model description}

Charm quarks are an excellent probe for the exploration of the medium created in ultrarelativistic heavy-ion collisions. Due to their high mass well above the medium temperature they are only produced in hard parton-parton collisions in the pre-equilibrium phase of heavy-ion collisions. In the following the charm quarks interact with the evolving medium and hadronize to D- and $\overline{\mathrm{D}}$ mesons. The decay products of these DD̄-mesons can finally be measured and help to shed light on the medium properties and the interaction of the D-mesons with the medium.

Moreover, D-mesons can be utilized to draw conclusions about the thermal QGP radiation. The correlated dileptons from DD̄-meson decays account for the overwhelming contribution of the hadron decay background in the invariant-mass range between the $\phi$ and $J / \psi$ peak of approximately $1 \mathrm{GeV}$ to $3 \mathrm{GeV}$. The dilepton radiation not originating from hadron decays can be accounted to thermal QGP radiation. Since the invariant mass spectrum of dilepton radiation at RHIC energy has been measured by PHENIX, the estimation of the hadron-decay background leads in turn to an estimation of the thermal QGP radiation.

In this letter we explore the invariant mass spectrum of dileptons from correlated DD̄-meson decays in $\mathrm{Au}+\mathrm{Au}$ collisions at $\sqrt{s_{N N}}=200 \mathrm{GeV}$. Since charm quarks are heavy particles, we can use a relativistic Langevin approach [1,2,3] for the propagation of charm quarks in the medium consisting of light particles. The required drag and diffusion coefficients are taken from a resonance approach [4], where the existence of D-meson resonances in the QGP is assumed. As background medium for this approach we utilize the UrQMD-hybrid model [5]. This model provides us with a realistic, well-tested medium evolution including event-by-event fluctuations.

The space-time production coordinates of the charm quarks are assigned based on a timeresolved "Glauber" approach utilizing the UrQMD model [6, 7]. We perform a first UrQMD run excluding interactions between the colliding nuclei and save the nucleon-nucleon space-time coordinates. These coordinates are used in a second, full UrQMD run as (possible) production spacetime coordinates for the charm quarks.

For the initial charm-quark momenta at RHIC energy we use $[1,8]$

$$
\frac{\mathrm{d} N}{\mathrm{~d} p_{T}}=\frac{C \cdot\left(1+A_{1} \cdot p_{T}^{2}\right)^{2} \cdot p_{T}}{\left(1+A_{2} \cdot p_{T}^{2}\right)^{A_{3}}},
$$

with $A_{1}=2.0 / \mathrm{GeV}^{2}, A_{2}=0.1471 / \mathrm{GeV}^{2}, A_{3}=21.0 . C$ is an arbitrary normalization constant with the unit $1 / \mathrm{GeV}^{2}$. Due to conservation laws the charm and anticharm quarks are produced in pairs and are emitted back-to-back.

Starting with these initial charm-quark distributions we perform the Langevin calculation in the hydrodynamic state of the medium evolution (cf. [8] for details).

The charm quarks decouple from the hot medium at a temperature of $130 \mathrm{MeV}$ and their hadronization is performed employing a coalescence mechanism [8]. The introduced Langevin model for charm-quark propagation has been successfully applied to the calculation of the medium modification factor $R_{A A}$ and the elliptic flow $v_{2}$ at FAIR, RHIC and LHC energies [8, 9, 10, 11]. 


\section{The invariant mass spectra of D-meson decays}

For the comparison of our approach to the invariant mass spectrum of dielectron pairs measured by PHENIX we assume three different thermalization scenarios:

No interaction: The charm quarks do not interact with the medium. However, their initial back-toback correlation is modified due to their hadronization via the coalescence mechanism.

Physical thermalization: We use the physical drag and diffusion coefficients from the resonance model. Utilizing these coefficients a good agreement to data for the medium modification and elliptic flow of heavy quarks at RHIC and LHC has been reached [8, 9, 10].

Extreme thermalization: The drag and diffusion coefficients are multiplied by a factor of 20 and a nearly full thermalization of the charm quarks to the background medium is achieved.

Our results for a minimum bias calculation $\left(\sigma / \sigma_{\mathrm{tot}}=0 \%-92 \%\right)$ with the appropriate experimental cuts are shown in Figure 1 in comparison to the PHENIX measurements.

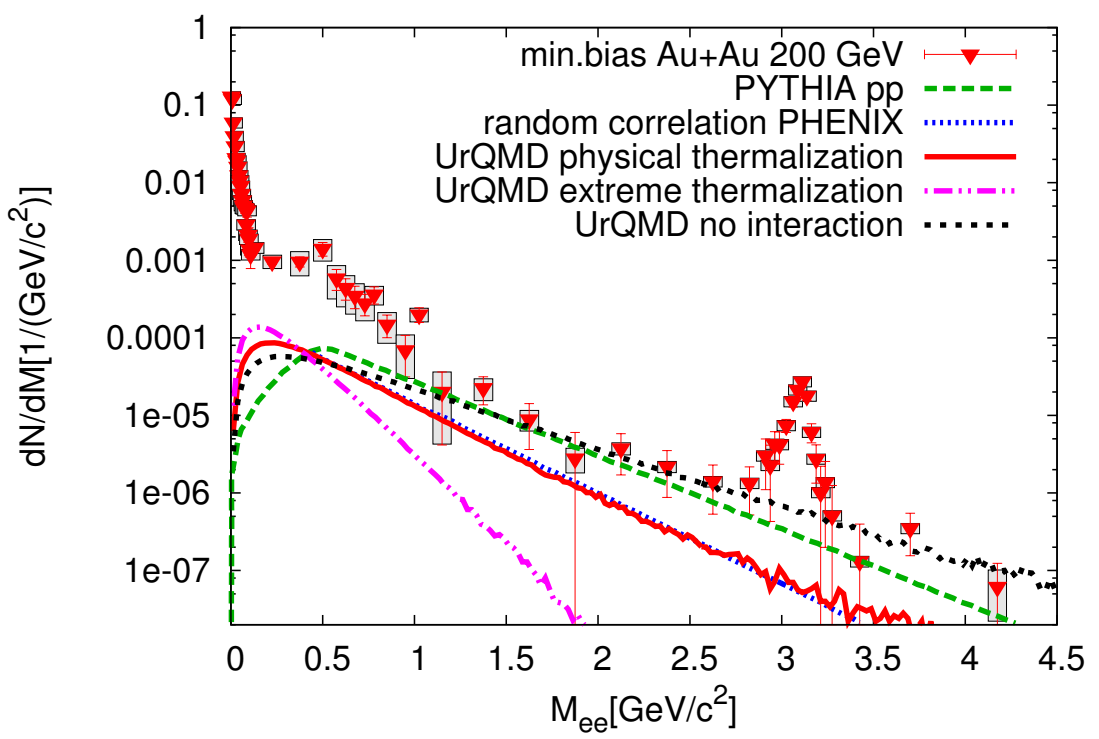

Figure 1: (Color online) Invariant mass spectra of electrons in $\mathrm{Au}+\mathrm{Au}$ collisions at $\sqrt{s}_{N N}=200 \mathrm{GeV}$ in the centrality range of $0-92 \%$ (min. bias). A rapidity cut of $|y|<0.35$ and a momentum cut of $p_{T}^{e}=0.2 \mathrm{GeV} / \mathrm{c}$ are applied. The dilepton data points are taken from a PHENIX measurement [12]. They are compared to calculations of electrons from D-meson decays. Also the pp calculation in PYTHIA and the random correlation calculation are taken from PHENIX [12]. The UrQMD calculations show three different scenarios: no interaction with the medium, physical drag and diffusion coefficients and extreme thermalization with 20 times higher coefficients. The difference between our physical scenario and the measured dilepton decays might be due to thermal radiation from the medium. The yields are normalized to the PYTHIA pp yield taken from [12].

The scenario without any medium interactions matches the data in the invariant mass range between 1 and $3 \mathrm{GeV}$ quite well. The calculation utilizing physical drag and diffusion coefficients results in a softer invariant mass spectrum. This leads to an underestimation of the data. This underestimation grows significantly with the invariant mass. The difference between this calculation 
and the data might be caused by thermal QGP radiation, which is expected to dominate among all other thermal sources in this invariant mass range. For the scenario with 20 -fold enhanced drag and diffusion coefficients a by far softer spectrum is produced and therefore the gap between our calculation and the measurements further increases.

The difference between our calculation neglecting interactions and the PYTHIA pp calculations stems from the differing initial charm-quark distribution and the inclusion of coalescence in our calculation. The discrepancy between our calculation assuming extreme thermalization and the PHENIX random correlation is caused by different assumptions for the random correlation. While PHENIX assumes a flat angular correlation, we assume a 3-dimensional random correlation function which corresponds to the geometrical $\Delta \Phi$ dependence (cf. [13] for details).

In summary, we have presented an estimation of the hadronic dielectron background originating in correlated DD̄-meson decay in the invariant mass region between 1 and $3 \mathrm{GeV}$. Our physical calculation suggests a significant contribution of thermal QGP radiation in this invariant mass range which might serve as a signal for the creation of a QGP.

\section{References}

[1] H. van Hees, M. Mannarelli, V. Greco and R. Rapp, Phys. Rev. Lett. 100 (2008) 192301 [arXiv:0709.2884 [hep-ph]].

[2] R. Rapp and H. van Hees, R. C. Hwa, X.-N. Wang (Ed.) Quark Gluon Plasma 4, World Scientific, 111 (2010) [arXiv:0903.1096 [hep-ph]].

[3] M. He, R. J. Fries and R. Rapp, Phys. Lett. B 701 (2011) 445 [arXiv:1103.6279 [nucl-th]].

[4] H. van Hees, V. Greco and R. Rapp, Phys. Rev. C 73 (2006) 034913 [nucl-th/0508055].

[5] H. Petersen, J. Steinheimer, G. Burau, M. Bleicher and H. Stocker, Phys. Rev. C 78 (2008) 044901 [arXiv:0806.1695 [nucl-th]].

[6] S. A. Bass, A. Dumitru, M. Bleicher, L. Bravina, E. Zabrodin, H. Stoecker and W. Greiner, Phys. Rev. C 60 (1999) 021902 [nucl-th/9902062].

[7] M. Bleicher, E. Zabrodin, C. Spieles, S. A. Bass, C. Ernst, S. Soff, L. Bravina and M. Belkacem et al., J. Phys. G 25 (1999) 1859 [hep-ph/9909407].

[8] T. Lang, H. van Hees, J. Steinheimer and M. Bleicher, arXiv:1211.6912 [hep-ph].

[9] T. Lang, H. van Hees, J. Steinheimer and M. Bleicher, arXiv:1208.1643 [hep-ph].

[10] T. Lang, H. van Hees, J. Steinheimer, Y. -P. Yan and M. Bleicher, J. Phys. Conf. Ser. 426 (2013) 012032 [arXiv:1212.0696 [hep-ph]].

[11] T. Lang, H. van Hees, J. Steinheimer and M. Bleicher, arXiv:1305.1797 [hep-ph].

[12] A. Adare et al. [PHENIX Collaboration], Phys. Rev. C 81 (2010) 034911 [arXiv:0912.0244 [nucl-ex]].

[13] T. Lang, H. van Hees, J. Steinheimer and M. Bleicher, arXiv:1305.7377 [hep-ph]. 